\title{
Effect of the Plan for Educating and Training Excellent Engineers on the Experimental Technical Ability of Undergraduate
}

\author{
X.D. Yuan \\ Materials Science and Engineering Institute \\ Shandong Jianzhu University \\ Jinan, Shandong, China
}

\author{
X.J. Yang \\ Shandong Product Quality Inspection Research Institute \\ Ji’nan, 250100, China \\ Shandong Key Laboratory for Testing Technology of Material \\ Chemical Safety \\ Ji’nan, 250103, China
}

\author{
G.L. Yuan \\ Jinan Engineering Quality and Work Safety Supervision Station \\ Ji’nan, Shandong, 250013, China
}

\begin{abstract}
The training system of 1234 engineering and technical personnel was implemented in requirements of the plan for educating and training excellent engineers by School of materials science and engineering, Shandong Jianzhu University. The construction of three-dimensional platform between the laboratory, the enterprise and the industry was strengthen and the experiment technical ability of undergraduate was improved by using of teaching tutorial and ideological mentor of advantage resources. The influence of 1234 training system of engineering and technical personnel on the experiment technical ability of undergraduates was studied taking the study on friction and wear properties of PTFE coatings as an example.
\end{abstract} wear

Keywords-experiment teaching; outstanding engineers; PTFE;

\section{INTRODUCTION}

The plan for educating and training excellent engineers (referred to as "excellence program"), is a major reform project implementing the "national medium and long-term educational reform and development plan (2010-2020)" and "national long-term talent development planning outline (2010-2020)". The pilot colleges and universities in the country draw up training objective of the engineering and technical personnel of the school combined with the school characteristic and the talent training standards under the guidance of general standards, since the beginning of June 23, 2010. Studies, including the teaching

reform of school and professional, personnel training mode, school enterprise cooperation and the reform of teaching practice were carried out by some colleges and universities in the "excellence program" requirements [1-8]. Material forming and control engineering in school of materials science and engineering is approved by the 2013 third batch of "excellence program" organized by the ministry of education. In requirement the training program of professional, studies about the demand for technical personnel materials engineering in the new period was carried out combing the characteristics of the material science itself by Shandong Jianzhu University. 1234 Training system of engineering and technical personnel was implemented And the experiment technical ability of undergraduates was improved. The so called talent training system of 1234 engineering technology includes the following four aspects: improving engineering practice ability of college students, the introduction of Double Tutorial System, three platforms of the industry, enterprise and laboratory, and four stages referring to tamp foundation, internship, painstaking research and independent innovation.

\section{TRAINING SYSTEM}

The influence of 1234 training system of engineering and technical personnel on the experiment technical ability of undergraduates was studied in this paper taking the study on the friction and wear properties of PTFE coatings as an example.

Training system of the engineering and technical personnel is training system by which engineering practical ability of students can be improved

The freshman makes efforts to learn theoretical knowledge, and consolidates the foundation. The sophomore experiences "Internship", understands the needs of enterprises. The junior participates or declares laboratory research projects, participates in the international conference and all kinds of associations of industry, innovates in thinking, and researches with great concentration. The senior carries out the work of graduation design combining engineering technical problems of enterprises, solves problems independently, and realizes the independent innovation. The implementation mode was shown in Figure 1. 




FIGURE I. TRAINING SYSTEM OF THE ENGINEERING AND TECHNICAL PERSONNEL.

\section{EXPERIMENTS}

\section{A. The Design of Friction and Wear Experiment of PTFE} Coatings

Polytetrafluoroethylene (PTFE) is a popular polymer solid lubricant due to its resistance to chemical attack in a wide variety of solvents and solutions, high melting point, low coefficient of friction, and biocompatibility. It is widely used in bearing and seals applications [9]. The machine parts experience early failure and leakage problem because of poor wear and abrasion resistance of PTFE [10-11]. To minimize the problem, various suitable fillers were added to PTFE.

The friction and wear properties of PTFE coatings were investigated in this paper. And the experiment parameters are as follows:

The friction and wear behavior of PTFE coatings was carried out on a ball-on-disk wear tester under vacuum conditions $\left(10^{-4} \mathrm{~Pa}\right)$. All PTFE coatings were slid against a GCr15-bearing steel ball. All sliding tests were carried out at a linear velocity from 0.2 to $2.4 \mathrm{~m} / \mathrm{s}$, at the load of $6 \mathrm{~N}$, and the distance of $1000 \mathrm{~m}$. In this work, three samples were tested at each condition. The friction coefficient and wear were the average values of these tests for each condition.

\section{B. Characterization of Friction and Wear Performance of PTFE Coatings}

The friction coefficient and wear of PTFE coatings under different sliding velocity are shown in figures 2 and 3, respectively. It can be seen from figure 2 that the friction coefficients of PTFE coatings under vacuum and air conditions are similar to each other and have similar trend curves. It also can be observed that the friction coefficients of the PTFE coatings first increase with the increase of sliding velocity and then decrease with the increase of sliding velocity when the sliding velocity is higher than $1.2 \mathrm{~m} / \mathrm{s}$. The variation of wear with sliding speed under the vacuum condition is shown in Figure3. The result shows that the wear of the PTFE coating first decreases with the increase of sliding speed and then increases as the sliding speed increases.

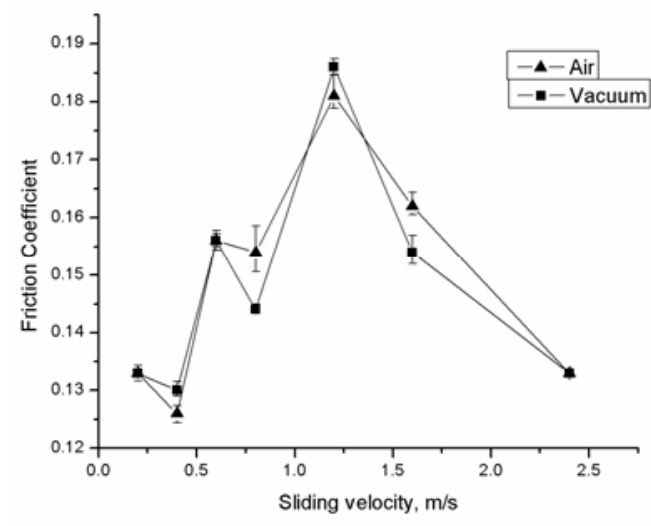

FIGURE II. VARIATIONS OF FRICTION COEFFICIENT WITH SLIDING VELOCITY (LOAD, 6N).

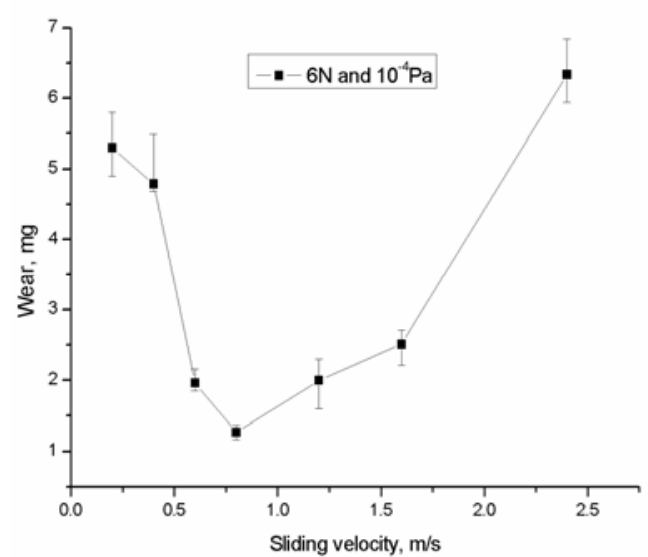

FIGURE III. VARIATIONS OF WEAR WITH SLIDING VELOCITY (LOAD, 6N AND PRESSURE, 10-4PA).

\section{CONCLUSIONS}

The 1234 training system of engineering and technical personnel was implemented by School of materials science and engineering of Shandong Jianzhu University. And the experiment technical ability of the undergraduate was improved

\section{ACKNOWLEDGEMENT}

Sponsored by the Research Fund for the College science and technology plan of Shandong Province ( Project No. J12LA11).

\section{REFERENCE}

[1] Huijuan Fu, Yong Xin. Pursuing “Engineer Excellence Program” to cultivate practical talents of technical engineering. Experimental technology and management, 2011, 28(11): 155-158.

[2] Dong Chen. Research on teaching reform of steel structure course based on outstanding engineers plans [J]. Journal of Chongqing university of science and technology (social sciences edition), 2011(6): 174-176.

[3] Lili Huang, Haisheng Zhang, Xiaohu Huang. Study on Educational Administration based on "Plan for Educating and Training Outstanding Engineers"[J].The 3rd International Annual Conference on Teaching Management and Curriculum Construction, 2012(5): 178-180.

[4] Yuhua Dai, Kai Gu, Jianping Huang. Development of an Enterprise Training Program for A Plan for Educating and Training Outstanding 
Engineers [J].Research and exploration in laboratory, 2012,31(12): 159-162.

[5] Wensong Lin, Liang He, Yanhui Liu. Experimental teaching reform of engineering materials course based on "plan for educating and training outstanding engineers" [J].Experimental technology and management, 2012,29(12): 20-22.

[6] Qisheng $\mathrm{Wu}$, Changsen Zhang, Baoxiang Jiao et al..The research of training mode for excellent engineer plan [J]. Chemical engineering education,2012(3):14-18.

[7] Shuangqing Lv, Yu Zhang. Research of software professional training plan Based on the "outstanding engineers plans" [J]. Vocational technology, 2011(7):132.

[8] Zhenzhong Sun, Shenggui Chen, Shouyan Zhong. Training mode of enterprise engineering practice for outstanding engineers[J].Research and exploration in laboratory, 2012,31(8): 285-289.

[9] W. Gregory Sawyer, Kevin D. Freudenberg, Praveen Bhimaraj, Linda S. Schadler, A study on the friction and wear behavior of PTFE filled with alumina nanoparticles[J]. Wear, 2003 (254) 573-580.

[10] D. Gong, Q. Xue, H. Wang, Study of the wear of filled polytetrafluoroethylene[J]. Wear, 1989 (134) 283-295.

[11] T.A. Blanchet, F.E. Kennedy, Sliding wear mechanism of (PTFE) and PTFE composites[J]. Wear, 1992 (152) 229-243. 